\title{
Macrophages Mediate the Anti-Tumor Effects of the Oncolytic Virus HSV1716 in Mammary Tumors
}

Amy Kwan ${ }^{1}$, Natalie Winder ${ }^{1}$, Emer Atkinson ${ }^{1}$, Haider Al-Janabi ${ }^{1}$, Richard J Allen ${ }^{1}$, Russell Hughes ${ }^{1}$, Mohammed Moamin ${ }^{1}$, Rikah Louie ${ }^{1}$, Dhanajay Evans ${ }^{1}$, Matthew Hutchinson ${ }^{1}$, Drew Capper ${ }^{1}$, Katie Cox ${ }^{1}$, Joshua Handley ${ }^{1}$, Adam Wilshaw ${ }^{1}$, Taewoo Kim ${ }^{1}$, Simon J Tazzyman $^{1}$, Sanjay Srivastava ${ }^{2}$, Penelope Ottewell ${ }^{1}$, Jayakumar Vadakekolathu ${ }^{3,4}$, Graham Pockley $^{3,4}$, Claire E Lewis ${ }^{1,5}$, Janet E Brown ${ }^{1,5}$, Sarah J Danson ${ }^{1,5}$, Joe Conner ${ }^{6}$, Munitta Muthana $^{1,5}$

${ }^{1}$ Department of Oncology and Metabolism, University of Sheffield Medical School, Sheffield, S10 2RX, UK.

${ }^{2}$ Department of Immunotherapeutics and Biotechnology and Center for Tumor Immunology and Targeted Cancer Therapy, Texas Tech University Health Sciences Center, Abilene, Texas, 79601, USA

${ }^{3}$ John van Geest Cancer Research Centre, School of Science and Technology, Nottingham Trent University, Nottingham, NG11 8NS, UK

${ }^{4}$ Centre for Health and Understanding Disease (CHAUD), School of Science and Technology, Nottingham Trent University, Nottingham, NG11 8NS, UK

${ }^{5}$ Sheffield ECMC, Cancer Clinical Trials Centre, Weston Park Hospital, Sheffield, S10 2SJ, UK

${ }^{6}$ Virttu Biologics Ltd, BioCity Scotland, Bo'ness Rd, Newhouse, ML1 5UH, UK

Running Title: Oncolytic virus reprograms macrophages within the TME

Key words: macrophages, breast, oncolytic viruses, PCNA

\section{Corresponding Author:}

Dr Munitta Muthana

Department of Oncology and Metabolism

University of Sheffield Medical School

Beech Hill Road, Sheffield, S10 2RX, UK

Tel: + 441142159057

Fax: + 441142713314

Email:m.muthana@sheffield.ac.uk

Conflict of interest disclosure statement: J. Conner is an employee of Virttu Biologics/Sorrento Therapeutics who own HSV1716.

Abstract 247

Word count: 5735 . Reference count: 50 . Figure count: 7. Supplementary Figures and Tables: 11 
Financial information:

- Cancer Research UK (CRUK grant reference: C25574/A24321)

- Sheffield Teaching Hospitals (grant reference: 12053)

- Team Verrico (grant reference: MS/149394)

- European Union's Horizon 2020 (Marie Sklodowska-Curie grant (872860PRISAR2) 


\section{Abstract}

2 Oncolytic viruses (OV) have been shown to activate the anti-tumor functions of specific 3 immune cells like $T$ cells. Here, we show OV can also reprogram TAMs to a less 4 immunosuppressive phenotype. Syngeneic, immunocompetent mouse models of primary 5 breast cancer were established using PyMT-TS1, 4T1 and E0771 cell lines and a metastatic 6 model of breast cancer was established using the 4T1 cell line. Tumor growth and overall 7 survival was assessed following intravenous administration of the OV, HSV1716 (a modified 8 herpes simplex virus). Infiltration and function of various immune effector cells was assessed 9 by NanoString, flow cytometry of dispersed tumors and immunofluorescence analysis of tumor sections. HSV1716 administration led to marked tumor shrinkage in primary mammary tumors and a decrease in metastases. This was associated with a significant increase in the recruitment/activation of cytotoxic $T$ cells, a reduction in the presence of regulatory $T$ cells and the reprograming of TAMs towards a pro-inflammatory, less immunosuppressive phenotype. These findings were supported by in vitro data demonstrating that human monocyte-derived macrophages (MDMs) host HSV1716 replication, and that this led to immunogenic macrophage lysis. These events were dependent on macrophage expression of proliferating cell nuclear antigen (PCNA). Finally, the anti-tumor effect of OV was markedly diminished when TAMs were depleted using clodronate liposomes. Together, our results show that TAMs play an essential role in support of the tumoricidal effect of the OV, HSV1716 - they both host viral replication via a novel, PCNA-dependent mechanism and are reprogramed to express a less immunosuppressive phenotype. 
2 Although modulating the immune system to target cancer has been a successful treatment for some solid malignancies, various forms of breast cancer are immunogenically cold [1] in that they exhibit a decreased mutational load and neoantigen expression. This leads to a lower infiltration by activated cytotoxic $T$ cells and is often accompanied by a highly immunosuppressive tumor microenvironment (TME) resulting in an intrinsic resistance to immunotherapies. The TME consists of cancer cells, tumor vasculature, fibroblasts, mesenchymal stem cells, adipocytes, extracellular matrix and immune system elements such as lymphocytes and tumor-associated macrophages (TAMs). TAMs are a key component of the TME that contribute to immune evasion, suppress lymphocyte activity and support tumor growth [2,3]. In particular, the accumulation of perivascular M2-skewed TAMs on the abluminal surface of tumor blood vessels has been shown to drive tumor relapse following radiotherapy [4] and chemotherapy [5], perhaps contributing to the eventual resistance of these standards of care $[5,6]$. A shift in the composition of the TME, together, with a burst in the release of tumor antigens, may turn a 'cold' tumor into a 'hot' one and therefore allow the host's immune system to recognise and halt tumor growth and metastasis [7].

Oncolytic viruses (OV) are a promising class of anti-cancer therapeutics which replicate in malignant cells and stimulate anti-tumor responses by initiating immunogenic cancer cell death (ICD), activating $\mathrm{T}$ cells and inducing protective anti-tumor immunity. Preclinical and early phase clinical studies, in a number of solid tumor types including breast cancer have shown OV to have therapeutic efficacy with minimal toxicity [8-10].

HSV1716 is an OV derived from the Herpes Simplex Virus HSV-1 strain 17. It possesses a deletion in the RL1 genes encoding ICP34.5. Mutants lacking ICP34.5 are selectively replication competent in cancer cells. The subsequent lysis of these cells induces anti-tumor immune responses both directly, through cell lysis, and indirectly, via the induction of 
1 immunogenic cell death and stimulation of adaptive immunity [11]. As HSV1716 maintains

2 expression of thymidine kinase, its toxicity is reversible by administering the anti-viral acyclovir, thereby providing a "therapeutic safety net" to clinical toxicity. Phase $\mathrm{I} / \mathrm{Il}$ trials in over 100 paediatric and adult patients with solid malignancies have demonstrated minimal systemic toxicity when HSV1716 is administered intratumorally (IT), intravenously (IV) or loco-regionally $[12,13]$.

Here, we show that HSV1716 effectively reduces primary and metastatic mouse breast tumors in vivo, in part, by replicating within and reprograming macrophages in the TME.

\section{Materials and Methods}

\section{Cell lines and culture}

Murine PyMT-TS1 [14] (a kind gift from Prof Johanna Joyce, Memorial Sloan Kettering Cancer Center (MSKCC), USA), E0771 (obtained from Dr Jessalyin Ubellacker (Harvard University, USA) and LUC-4T1-BR [15] (obtained from Prof Sanjay Srivastava, University of Texas, USA) mammary cancer cells were used in vivo. Human MCF-7, MDA-MB-231, MCF10DCIS and SKBR3 cells, murine 4T1, EO771 and PyMT-TS1 cells and African Green Monkey Vero cell lines were used in vitro. Unless specified, all cell lines were purchased from the ATCC between 2015-2018 and used within 30 passages. Murine E0771 and human MDA-MB-231 cells were maintained in RPMI supplemented with $10 \% \mathrm{v} / \mathrm{v}$ fetal bovine serum (FBS) and 1\% L-glutamine. Murine Luc-4T1-BR cells (4T1 cells transfected to express luciferase) were maintained in Dulbecco's Modified Eagle's Medium (DMEM) supplemented with $10 \% \mathrm{v} / \mathrm{v}$ FBS. All cells were used within 20 passages and were cultured at $37^{\circ} \mathrm{C}$ in $5 \%$ $\mathrm{v} / \mathrm{v} \mathrm{CO}_{2}$. The identities of all cell lines were regularly confirmed using microsatellite analysis and were tested to be mycoplasma free. All culture reagents were purchased from Lonzo BioWhittaker Ltd. 
1 Preparation of monocyte-derived macrophages (MDMs)

2 Human monocytes were isolated from mononuclear cells derived from human buffy coats

3

4

5

6

7

8

9 obtained from the NHS Blood and Transplant Unit, Sheffield, as previously described [16]. Briefly, the peripheral blood mononuclear cell (PBMC) layer was collected following centrifugation over Ficoll (Sigma-Aldrich, UK) and seeded overnight in Iscove's Modified

Dulbecco's Medium (IMDM, Sigma-Aldrich, UK) and $2 \% \mathrm{v} / \mathrm{v}$ human $A B$ serum (Sigma Aldrich, UK). Non-adherent cells were removed, and macrophages allowed to fully differentiate.

\section{Virus production and handling}

HSV1716 (unlabelled) and HSV1716-GFP (in which GFP is driven by a CMV promoter) were obtained from Virttu Biologics (Glasgow, UK) in stocks of $1 \times 10^{8}$ Particle Forming Units (PFU) in compound sodium lactate (Hartmann's solution) with $10 \% \mathrm{v} / \mathrm{v}$ glycerol. All vials were stored at $-80^{\circ} \mathrm{C}$ and freshly thawed on ice immediately before each experiment.

\section{HSV1716 infection of MDMs in vitro}

MDMs were washed in phosphate buffered saline (PBS), suspended in 500 $\mathrm{LL}$ serum-free RPMI medium and then incubated with HSV1716 virus (MOI 5) for 2 hours at $37^{\circ} \mathrm{C}$ in $5 \% \mathrm{v} / \mathrm{v}$ $\mathrm{CO}_{2}$. Non-infected virus was washed off and cells were analysed 24-72 hours post infection. For plaque assays supernatants were removed at each time point and added to Vero cells as described below.

\section{Mixed lymphocyte reaction (MLR)}

A Mixed Lymphocyte Reaction (MLR) was used to identify leukocyte activation and proliferation in an autologous reaction. MDMs were obtained from human buffy coats and cultured as above. Lymphocytes, from the same donor, were frozen down in $90 \% \mathrm{v} / \mathrm{v}$ FBS $+10 \%$ v/v dimethyl sulfoxide (DMSO) until needed. Once mature, MDMs alone or MDMs infected with HSV1716 for 4 hours were co-incubated with lymphocytes at a ratio of 1:6, as 
1 described previously [17]. MDA-MB-231 cells were also added, if needed, at a 1:1 ratio with

2 MDMs. Lymphocytes were co-cultured with macrophages for 24 hours before analysis by

3 flow cytometry.

4

$5 \quad$ Flow cytometric analysis

6 MDMs (24 hours after infection) and dissociated mammary tumors were stained with

7 fluorescent antibodies (Supplementary Table S1) [5]. All antibody incubations were

8 performed for 1 hour at $4^{\circ} \mathrm{C}$ and the samples were analysed using an BD ${ }^{\mathrm{TM}}$ LSR II flow

9 cytometer (BD Biosciences, Franklin Lakes, NJ, USA) and data analyzed processed using

10 FlowJo $^{\mathrm{TM}}$ Flow Cytometric Data Analysis Software (BD Biosciences). The mouse immune

11 cell populations analysed included: neutrophils $\left(C D 45^{+} C D 11 b^{+} L y 6 G^{+}\right)$, monocytes

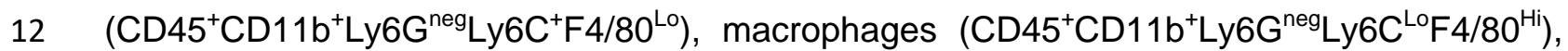

$13 \mathrm{~T}_{\text {Helper }}\left(\mathrm{CD} 45^{+} \mathrm{CD} 3^{+} \mathrm{CD} 4^{+}\right)$and cytotoxic T-cells $\left(\mathrm{CD} 45^{+} \mathrm{CD} 3^{+} \mathrm{CD} 8^{+}\right)$. The membrane

14 impermeant, fixable, amine reactive dye Zombie UV TM Fixable (BioLegend Inc., San Diego,

15 CA, USA) was used to discriminate between live and dead cells. All data are presented as

16 the proportion of viable leukocytes.

Real-Time PCR (qPCR)

Total mRNA was extracted from cultured MDMs or murine tissues using the RNeasy ${ }^{\mathrm{TM}}$ Mini Kit (Qiagen). A list of primer sequences is given in Supplementary Table S2. The Ct values generated from these samples were normalized to a housekeeping gene. Relative gene expression to untreated macrophages was estimated via normalisation of the gene of interest to the housekeeping gene followed by the comparative Ct $\left(2^{-\Delta \Delta C t}\right)$ method.

\section{Western blot analysis}

Protein detection by SDS-PAGE was carried out on MDMs [18]. Protein samples were denatured at $70^{\circ} \mathrm{C}$ and loaded onto a gel with Laemmli sample buffer. The gel was transferred onto a nitrocellulose membrane (Invitrogen) using an iBlot gel transfer device. 
1 The membrane was blocked in $5 \% \mathrm{v} / \mathrm{v}$ milk for an hour and incubated, at room temperature,

2 with primary antibodies for 90 minutes and secondary antibodies for an hour. Membranes were probed with enhanced chemiluminescence (ECL) (BIO-RAD) and visualised using a Chemidoc 2011 (BIO-RAD).

\section{HSV1716-induced cell lysis}

To assess cell lysis induced by HSV1716, various cell lines were seeded at $1 \times 10^{5}$ cells/well in a 24 well plate and infected with HSV1716 at MOI 5 (unless otherwise stated). At 24 and 48 hours, cells were stained with $2 \mu \mathrm{g} / \mathrm{ml}$ propidium iodide (PI). Cell numbers and PI positivity were analysed on the $\mathrm{BD}^{\mathrm{TM}}$ LRSII flow cytometer (Thermo Fischer Scientific). FlowJo ${ }^{\mathrm{TM}}$ software was used to analyse cell death based on a change in fluorescence against FL3-H for $\mathrm{PI}$ and FL1-H for GFP expression.

\section{Viral replication}

qPCR: RNA was isolated from infected MDMs using the RNeasy Mini Kit (Qiagen) followed by cDNA synthesis using SuperScript ${ }^{\mathrm{TM}}$ III Reverse Transcriptase (Life Technologies, Paisley, UK). cDNA was analyzed using viral replication genes ICPO, ICP8 and gB with GAPDH as the housekeeping gene using SYBR ${ }^{\text {тм }}$ Green (Primer Design, Chandler's Ford, UK) (see Supplementary Table S2).

In vivo viral detection: Immunohistochemistry was carried out on fixed tissue sections stained using a polyclonal sheep HSV antibody at a dilution of 1:500 (kind gift from Virttu Biologics) for 1 hour. Staining was then visualized using a sheep VECTASTAIN ${ }^{\text {TM }}$ ABC HRP (Horseradish Peroxidase) Kit (Vector Laboratories, Peterborough, UK). Slides were counter stained, mounted and sections scanned using Hamamatsu NanoZoomer XR (Hamamatsu, Hertfordshire, UK). 
1 Plaque assays: These were performed as described previously by Baer and Kehn-Hall [19].

2 Briefly, confluent monolayers of Vero cells were inoculated with serial dilutions of supernatants derived from infected macrophages as described above. After 2 hours, supernatants were removed and monolayers were overlaid with $4 \% \mathrm{w} / \mathrm{v}$ agarose:culture medium (1:10) which was allowed to solidify for 15 minutes at room temperature before incubation in a humidified incubator for 72 hours at $37^{\circ} \mathrm{C}$. Paraformaldehyde $(4 \% \mathrm{w} / \mathrm{v})$ was applied to agarose plugs for 1 hour to fix the cell monolayers before their removal. Cells were washed with PBS, stained with $1 \mathrm{ml}$ crystal violet for 5 minutes and rinsed with tap water. Once dried, plaques were counted per well and viral titre determined.

\section{Analysis of MDM death}

To understand how HSV1716 mediated the oncolysis of MDMs in vitro, the expression of a panel of cell death markers was determined using qPCR. cDNA was analyzed using a predesigned apoptosis and survival array (tier 1) (BIO-RAD, Hertfordshire, UK) and then validated by qPCR using a panel of apoptotic genes (FASL and BCL2), autophagy genes (ATG5 and LC3B) and ICD genes (HMGB1 and CalR), with GAPDH as the housekeeping gene (see Supplementary Table S2). Analysis using a HMGB1 ELISA Kit II (Shino-Test, Kanagawa, Japan) and an ENLITEN ATP assay (Promega, Madison, WI, USA) confirmed the presence of immunogenic cell death (ICD).

\section{Knockdown of PCNA expression in MDMs}

MDMs were transfected with PCNA or nonspecific siRNAs (Accell Human PCNA siRNA SMARTpool, 10nmol, Thermo Scientific/Dharmacon). For this, MDMs were aliquoted into a 6 -well plate $\left(0.5 \times 10^{5}\right.$ macrophages/well $)$ and incubated with $1.5 \mathrm{ml}$ of Accell delivery medium containing $1 \mu \mathrm{M}$ siRNA, after which they were incubated at $37^{\circ} \mathrm{C}$ for 72 hours. Protein or mRNA knockdowns were confirmed by Western blot and qPCR and viability determined using the MTS CellTiter 96 ${ }^{\mathrm{TM}}$ AQueous One Solution Cell Proliferation Assay (Promega). 
2 Animal procedures were carried out in accordance with the UK Animals (Scientific

Procedures) Act 1986 with approval from the UK Home Office approval (PPL70/8670), the ARRIVE (Animal Research: Reporting of In Vivo Experiments) guidelines and the University of Sheffield Animal Welfare Ethical Review Body (AWERB). All female mice were obtained from Charles River Laboratory at 6-8 weeks and acclimatised in the Biological Services Laboratory for 7 days prior to experimentation. Animals were anesthetised using $3-4 \%$ isofluorane in $70: 30 \% \mathrm{~N}_{2} \mathrm{O}: \mathrm{O}_{2}$.

Orthotopic mammary tumor model: Mammary cancer cells $\left(1 \times 10^{6}\right.$ PyMT TS1 cells in $50 \mu$ of 1:1 Matrigel $\left.^{\mathrm{TM}}\right)$, were implanted into the $4^{\text {th }}$ mammary fat pads of $6-7$ week old syngeneic FVB mice ( $n=10 /$ group). E0771 and 4T1 cells were implanted via intraductal injection to the mammary fat pads of syngeneic C57BL/6 and BALB/c mice respectively. Mammary tumor growth was assessed by digital calliper measurement every 2-3 days and when tumors reached $150 \mathrm{~mm}^{3}$, mice were randomly divided into groups and received a single $100 \mu \mathrm{l}$ injection of PBS or HSV1716 ( $1 \times 10^{6}$ PFU) intravenously via tail vein injection. Of note, a similar treatment schedule, was performed to compare intravenous (IV) and intratumoral (IT) injections in the PyMT TS1 model. Of note, mice in the PBS groups became unwell at day 9 and therefore some mice, in both groups, were culled early for post-mortem comparison of tissues. Excised tissues (tumors, brain, liver, lungs, kidney and spleen) were embedded in OCT freezing media or paraffin wax for immunocytochemical and histological labeling studies. Tumors were dispersed by enzymatic digestion after first dicing into approximately $1 \mathrm{~mm}^{3}$ pieces. These pieces were incubated for 40 minutes at $37^{\circ} \mathrm{C}$ in serum-free IMDM (VWR International, PA, USA) supplemented with $2 \mathrm{mg} / \mathrm{mL}$ dispase, $0.2 \mathrm{mg} / \mathrm{mL}$ collagenase IV (Sigma Aldrich, St. Louis, MO, USA) and 100U/mL DNase (Merck Millipore, Burlington, MA, USA). Dispersed tumors were passed through $70 \mu \mathrm{m}$ nylon filters (Becton Dickinson, Franklin Lakes, NJ, USA) and maintained on ice in PBS or cryo-preserved in $90 \% \mathrm{v} / \mathrm{v}$ FBS and $10 \% \mathrm{v} / \mathrm{v}$ DMSO for flow cytometric analysis. 
1 Experimental metastasis model: To model the metastatic seeding seen in breast cancer

2 patients, we used a metastatic $4 \mathrm{~T} 1$ cell line which metastasises to brain, lung, liver and

3

4

5

6

7

8

9 bones when administered via intracardiac route[15]. For this model, $1 \times 10^{5}$ LUC-4T1-BR cells were filtered and injected into the left ventricle of 6-7-week-old female BALB/c mice. Tumors were allowed to grow for 5 days following which animals were randomly allocated $(n=6$ per group) and received either PBS, 1 dose of HSV1716 $\left(1 \times 10^{7}\right)$ or 3 doses of HSV1716 $\left(1 \times 10^{7}\right.$ given on day 1, 3 and 5). Animals were imaged 2-3 times a week using a luminescence in vivo imaging system (IVIS Lumina II imaging, Caliper Life Sciences) following intraperitoneal injection of luciferin $(150 \mathrm{mg} / \mathrm{kg})$. Mice were sacrificed if they reached a humane end point (weight loss over 20\%, signs of distress (e.g. breathlessness or pain) or the experimental end point of 50 days following tumour inoculation. Weight loss was the most common cause of premature sacrifice.

Macrophage depletion model: Macrophages were depleted using a single dose of clodronate liposomes (CL) (Liposoma B.V.) 48 hours prior to viral administration in the primary mammary model. When mammary tumors reached $150 \mathrm{~mm}^{3}$, animals were divided into 4 groups, $n=5 /$ group. Control groups received either 1 dose of PBS $(100 \mu l)$ or 1 dose of clodronate liposomes $(100 \mu \mathrm{l})$. Treatment groups received an intravenous injection of HSV1716 (1 dose, $1 \times 10^{6} \mathrm{PFU}$ ) or intravenous injection of CL followed by intravenous HSV1716 (1 dose, $1 \times 10^{6}$ PFU). Animals were monitored and primary tumors measured every 2-3 days using callipers. All animals were culled as soon as one animal had a tumor of $800 \mathrm{~mm}^{3}$.

\section{Tissue Analysis}

Frozen tumor sections were blocked with $1 \% \mathrm{w} / \mathrm{v}$ BSA and $5 \% \mathrm{v} / \mathrm{v}$ goat serum for 30 minutes and incubated, at room temperature, with the relevant primary antibodies (Supplementary Table S1), for an hour. Alexa Fluor ${ }^{\mathrm{TM}}$-conjugated anti-rabbit or anti-rat secondary antibodies (as appropriate) were used to detect primary antibody binding. Nuclei in all tumor sections 
1 were counterstained with DAPI. Slides were visualised using a Nikon DualCam system

2 microscope, a Nikon A1 Confocal Laser Microscope and an EVOS ${ }^{\mathrm{TM}}$ Cell Imaging System

3 (ThermoFisher). Formalin-fixed, paraffin-embedded tissues were rehydrated, peroxidase

4 blocked, antigen retrieved, serum blocked, and then incubated with primary antibodies for 1-

52 hours. Primary antibodies were detected with ABC or Polymer detection kits followed by

6 chromogen staining with 3'-Diaminobenzidine (DAB). Following Haematoxylin and Eosin

7 staining, slides were visualized using the Hamamatsu NanoZoomer XR scanner

8 (Hamamatsu, Hertfordshire, UK) and staining in 5 randomly selected fields of view per tumor

9 quantified using ImageScope (Leica Biosystems).

\section{NanoString $\mathrm{nCounter}{ }^{\mathrm{TM}}$ gene expression analysis}

Amplification-free gene expression profiling of tumor tissue using a NanoString $\mathrm{nCounter}{ }^{\mathrm{TM}}$ FLEX platform and the murine PanCancer Immune Profiling Panel, which consist of 750 immune related genes and 20 housekeeping genes (NanoString Technologies Inc) was undertaken in the John van Geest Cancer Research Centre (Nottingham Trent University). Total mRNA was extracted from cultured MDMs or murine tissues using the RNeasy ${ }^{\mathrm{TM}}$ Mini Kit (Qiagen) and quality controlled using a NanoDrop ${ }^{\mathrm{TM}} 8000$ Spectrophotometer. For gene expression profiling, 150ng of total RNA from each sample was used for NanoString probe hybridisation which was undertaken overnight $\left(20\right.$ hours) at $65^{\circ} \mathrm{C}$ in a PCR machine with heated lid (each reaction mixture contains $5 \mu$ l of RNA solution (150ng), $8 \mu$ l of reporter probe and $2 \mu \mathrm{l}$ of capture probe). After overnight hybridization, excess probes were removed using the NanoString nCounter ${ }^{\mathrm{TM}}$ Prep Station and magnetic beads, hybridized mRNA/probe were immobilised on a streptavidin-coated cartridge. The processed cartridge was subsequently scanned, and raw data generated at high-resolution (555 fields of view, fov) using a NanoString nCounter ${ }^{\mathrm{TM}}$ digital analyser platform and processed using nSolver ${ }^{\mathrm{TM}}$ Data Analysis Software (V.4.0). Imaging quality control (QC), mRNA positive control QC and normalisation QC were checked, and all the samples were with the quality parameters of NanoString gene expression assays. Differential expression, pathway and cell type scoring 
1 was performed using the nSolver ${ }^{\mathrm{TM}}$ Advanced Analysis Module v.2.0.115. Data

2 normalization was performed using the geNorm algorithm for the selection of the best

3 housekeeping genes. Genes which showed $\geq 2$, fold change in their expression with a BY

4 (Benjamini-Yekutieli procedure) $\mathrm{P}$ value $\leq 0.05$ were considered significantly different

5 between the groups.

6

$7 \quad$ Apoptosis and pro-survival array

8 The Apoptosis and Pro-Survival Tier 1 array (BIO-RAD) was used to assess cell death. For

9 this, cDNA was synthesised from control or infected MDMs using the Precision nanoScript2 Reverse Transcription Kit (PrimerDesign). cDNA was plated into the 386 well qPCR plate and processed using an Applied Biosystems 7900 Real-Time PCR System.

\section{Statistics}

All statistical analyses were performed using GraphPad Prism 8 and the tests are described in the Figure legends. Data are means \pm SEM (as indicated) and $P$ values of $<0.05$ were considered statistically significant.

Results

\section{HSV1716 has anti-tumor activity in breast cancer models}

The susceptibility of breast cancer cells to HSV1716 infection (Supplementary Fig. S1A) and virus-mediated death (Supplementary Fig. S1B) was demonstrated in vitro using a panel of human and murine breast cancer cells lines (MCF7, MDA-MB-231, SKBR3, MCF10DCIS.com, 4T1, EO771, and PyMT-TS1).

The cytotoxic potential of HSV1716 was then assessed in three in vivo models of primary breast cancer. First, we investigated the best route of HSV1716 delivery in the PyMT-TS1 model. For this, animals were randomly assigned into one of 3 treatment groups; control (PBS), intratumoral (IT) HSV1716 and intravenous (IV) HSV1716, with a reduction in primary 
1 mammary tumor growth being observed in both the IT and IV groups (Supplementary Fig.

2 S2A). IT and IV administration of HSV1716 also reduced pulmonary metastasis and increased tumor necrosis (Supplementary Fig. S2B and S2C). Given the positive response to IV HSV1716, and that the IV route is currently the preferred modality to deliver breast cancer chemotherapies, the remainder of the study focused on IV delivery of HSV1716.

In three models of primary breast cancer (PyMT-TS1, E0771 and 4T1) we demonstrated IV HSV1716 to significantly slow the growth of orthotopically implanted tumors (Figure 1A). The number of subsequent spontaneous lung metastases in these animals was also significantly reduced in the IV treated groups (Figure 1B). Furthermore, we observed that early introduction of HSV1716 prevented the formation of breast cancer metastases and thereby increased overall survival in the metastatic 4T1 model. In this model, luciferase labelled 4T1 cells were injected into the left ventricle of the heart. Five days later, mice were treated with PBS, HSV1716 (1 dose) or HSV1716 (3 doses). Doses were repeated every 48 hours (Figure 2A). Tumor growth and spread were monitored using bioluminescent in vivo imaging 2-3 times weekly (Figure 2B). A survival advantage was seen in the HSV1716 treated groups over the PBS control, and a significant number of animals showed no sign of disease by day 50 (Figure 2C). As shown, this was more marked with repeated doses of the virus, in which instance, all mice survived to the experimental endpoint (50 days) compared to an average survival of 31 days for one dose and 24.5 days for PBS mice $(p=0.0002, C l 15.49$ 35.18). This may be because the immunomodulatory effects of the virus takes time to develop and repeated dosing allows for this to occur, or that repeat dosing does not allow circulating tumor cells or micro-metastases to develop. The presence of brain, liver and lung metastasis was assessed and a significant reduction in the number of lung metastases in the groups treated with HSV1716 observed, with a trend to less metastases in the brain and liver (Figure 2D). Together, these exciting results support the possible use of HSV1716 to treat breast cancer. 
2 The influence of HSV1716 treatment on the immune content of the TME, including immune 3 infiltrates was examined using the NanoString nCounter ${ }^{\mathrm{TM}}$ Mouse PanCancer Immune 4 Profiling Panel. This large targeted gene panel comprised of specific gene sets to 5 understand different immune cell types and their functions in the TME. As shown in Figure $63 \mathrm{~A}$ treatment with HSV1716 induced the differential regulation of 282 genes (where $p=0.05$ ).

7 The top 20 genes and their biological functions are highlighted in Supplementary Table 3, and these include genes involved in innate and adaptive immune responses (e.g. CD55, IL-

9 21, TXk, Thbs1), immune cell function (CD22, CD37, Blnk, Sell, CD247, IL7R, Dpp4, Btla, CD247, Thbs1) and the TNF pathway (TNFrsf13b, Ltb). Supplementary Fig. 3A shows genes related to upregulated pathways including innate and adaptive immunity, inflammation, cytokines and receptors, apoptosis and cell type scores (dendritic cell, natural killer, macrophages and $\mathrm{T}$ cells) that were significantly upregulated following intravenous HSV1716 (Supplementary Fig. 3B).

To confirm the presence of these immune cells in the TME, flow cytometric analysis of dispersed PyMT primary tumors demonstrated a significant increase in CD11 $\mathrm{b}^{+} \mathrm{Ly}_{6 \mathrm{C}^{+}}$ monocytes, $\mathrm{CD} 11 \mathrm{~b}^{+}, \mathrm{LY}_{6 \mathrm{G}}{ }^{+}$neutrophils, $\mathrm{CD}^{+} \mathrm{T}$ cells, and $\mathrm{CD} 8^{+}$cytotoxic $\mathrm{T}$ cells following HSV1716 treatment (Figure 3B). This is consistent with other published studies using oncolytic viruses [20]. Triple immunofluorescence analysis of tumor sections revealed an increase in activated $\mathrm{CD}^{+}$cytotoxic $\mathrm{T}$ cells (i.e. $\mathrm{FFNg}^{+}$or $\mathrm{PD}-1^{+} \mathrm{CD} 3^{+} \mathrm{CD} 8^{+}$cells) in this mouse model after HSV1716 treatment (Figure 3C). Furthermore, HSV1716 treatment led to a reduction in the number of $\mathrm{CD}^{+}{ }^{+} \mathrm{FOXP3}{ }^{+} \mathrm{T}$ regulatory (Treg) cells (Figure 3D).

\section{HSV1716 reprograms TAMs to a more pro-inflammatory and perivascular phenotype}

NanoString nCounter ${ }^{\mathrm{TM}}$ gene profiling revealed an upregulation in the macrophage function scores (Supplementary Fig. 3B). Despite this, we observed the average number of TAMs 
1 within tumor samples (\% of CD45+F4/80+ per 100,000 events of viable cells) was not

2 significantly altered following HSV1716 treatment (Figure 3B), but that there was a marked change in their phenotype. First, HSV1716 treatment significantly decreased the prevalence of "tumour promoting" perivascular macrophages (i.e. F4/80+ TAMs directly in contact with $\mathrm{CD} 1^{+}$endothelial cells) in PyMT mammary tumors (Figure 4A). Second, HSV1716 treatment significantly increased the number of $\mathrm{F} 4 / 80^{+}$TAMs expressing pro-inflammatory, M1-like markers, IL-12 and iNOS, relative to matched controls (no virus). Furthermore, HSV1716 treatment significantly reduced $(P<0.05)$ the number of $F 4 / 80^{+}$TAMs expressing the M2-like marker 'MRC1' (Figure 4B). This reprogramming of TAMs has the potential to change the balance in the TME, in that M2-like TAMs become M1-like, thereby promoting the recruitment of adaptive immune cells and cytotoxic potential.

Our in vitro experiments also demonstrated that human MDMs infected with HSV1716 undergo a transformation to a more inflammatory phenotype, specifically a greater expression of M1-like markers (CD80 $\left.{ }^{\text {hi }}, C D 86^{\text {hi }}, \mathrm{PD}-\mathrm{L} 1^{\mathrm{hi}}\right)$ and lower expression of M2-like markers $\left(C D 64^{10}, C D 163^{10}\right.$ and CD206/MRC1 $1^{10}$ ) (Supplementary Fig. S4A) and increases in the expression of pro-inflammatory vs. anti-inflammatory markers at the mRNA level (Supplementary Fig. S4B). Infected macrophages also secreted pro-inflammatory cytokines including IL-6, IL-12 and TNF $\alpha$ (Supplementary Fig. S4C) and increased levels of nitric oxide (Supplementary Fig. S4D).

\section{Macrophages support HSV1716 replication and undergo immunogenic cell death}

The observation that HSV1716 treatment led to a co-localisation of F4/80+ ${ }^{+}$AMs and HSV1716 in the TME (Figure 5A), prompted the question as to whether macrophages are permissive to HSV1716 infection and, if so, what is the nature of this relationship? To investigate this, MDMs derived from human buffy coats were infected with the HSV1716 for 2 hours, following which excess virus was washed off, cells incubated for a further 24 hours and infection confirmed on the basis of GFP expression using the reporter virus 
1 (HSV1716:GFP) (Figure 5B). Plaque assays determined viral titres within the supernatants

2 of these virally infected MDMs. An increase in viral titres at 24,48 and 72 hours was seen

3 following infection of the HSV permissive Vero cell line (Figure 5C). Furthermore, expression

4 of genes required for early (ICP0), mid (ICP8) and late (gB) viral replication was quantified

5 using qPCR (Figure 5D). These studies confirmed that HSV1716 went through all stages of

6 viral replication within MDMs in vitro and suggest that MDMs infected with HSV1716

7 supported active viral replication.

8

9 As we saw a significant drop in macrophage numbers 24 hours after treatment in vivo (Supplementary Fig. S5A), we next assessed the impact of HSV1716 on macrophage viability and sought to identify the cause of this in human MDMs in vitro. First, we noted that human MDMs infected with HSV1716 undergo enhanced levels of cell death compared to non-infected cells in vitro (Supplementary Fig. 5B). To ascertain the mechanism of this, a cell death array was performed (Supplementary Table 4). The mechanism of macrophage cell death and signalling pathways were largely apoptotic and immunogenic, with an increase in Fas ligand and HMGB1 expression, and a down regulation of HSP genes (validated by qPCR) (Supplementary Fig. S5C). These data were supported by Western blots showing an increased production of apoptotic proteins, FADD, FASL and caspase 3, (Supplementary Fig. S5D) and ELISA assays showing an increased secretion of immunogenic proteins, HMGB1 (Supplementary Fig. S5E) and extracellular ATP (Supplementary Fig. S5F).

\section{Proliferating cell nuclear antigen (PNCA) mediates HSV1716 replication in TAMs}

Oncolytic virus replication is known to occur within dividing tumor cells and therefore we were keen to determine why HSV1716 was replicating within macrophages (i.e. terminally differentiated cells). Wild type HSV has been shown to initiate proliferation in nonproliferative cells such as neurones by interacting with the cellular protein, PCNA [21], although this has not previously been demonstrated in macrophages. Interestingly, we found 
that HSV1716-infected PyMT-TS1 tumors contained significantly more $\mathrm{PCNA}^{+}$TAMs (Figure.6A).

PCNA expression in HSV1716 infected and non-infected MDMs was measured in vitro at both the gene and protein level. MDMs infected with HSV1716 exhibited significant relative increases in PCNA mRNA (11-fold increase, $p=0.0137)$ and protein expression $(p=0.0173)$ compared with untreated MDMs (Supplementary Fig. S6A). This increased expression was more marked when MDMs were infected with HSV1716 in the presence of tumorconditioned medium (TCM) (Figure. 6B). Given the increase in PCNA following infection we wanted to ascertain whether HSV1716 replication was possible in the absence of PCNA. PCNA knockdown (PCNA ${ }^{K D}$ ) was carried out using Accell siRNA (Supplementary Fig. S6B). This had no effect on viral infectivity and the viability of macrophages compared to the nontargeting control (Supplementary Fig. S6C and S6D). Indeed, in the absence of PCNA, HSV1716 was unable to undergo viral replication within infected macrophages. This is evidenced by the lack of cell death in MDA-MB-231 cells following incubation with supernatants taken from infected macrophage cultures following PCNA ${ }^{\mathrm{KD}}$ (Figure 6C) and a reduction in viral replication genes (Figure 6D). We therefore speculate that the presence of PCNA is likely the cause of viral replication and macrophage cell death after infection.

Given the interactions between HSV1716 and macrophages, we then investigated the role of macrophages in the anti-tumor responses seen with this oncolytic virus in our in vivo model. To explore this, circulating monocytes and macrophages were eliminated in vivo using clodronate liposomes [22] (Figure 7A). Clodronate liposomes induce the apoptosis of macrophages and others have shown that this to trigger anti-tumor activity by inducing changes in the TME [23]. We administered a single dose of clodronate at 48 hours prior to treatment with HSV1716 and noticed a modest reduction in macrophage number (control (PBS) $69.4+/-11.4$, clodronate alone $33.9+/-11.6, p=0.0001$. As expected, IV HSV1716 decreased tumor growth in comparison to PBS controls. However, this effect was lost when 
1 monocytes/macrophages were depleted, in that there was an increase in primary tumor

2 growth (Figure 7B) and a development of lung metastases when data for HSV1716

3 treatment are compared in the presence $(\mathrm{OV}+\mathrm{CL})$ or absence $(\mathrm{OV})$ of clodronate liposomes

4 for simplicity (Figure 7C). Of note, a greater number of lung metastases were seen in this

5 experiment compared to the data presented in Figure 1B. This is likely due to the endpoint in

6 this experiment being later (day 14) as opposed to day 9 in Figure 1B. T cell subsets in

7 these two groups were examined using immunofluorescence post-mortem. An increase in

8 the number of $\mathrm{CD} 4^{+} \mathrm{T}$ cells and a decrease in $\mathrm{CD}^{+} \mathrm{T}$ cells were seen in animals in which

9 macrophages had been eliminated (Figure 7D). These data suggest that TAMs are key to the cytotoxicity of HSV1716 infection in vivo and may mediate this through their regulation of $T$ cell subsets in the TME. The interaction between macrophages and $T$ cells was also confirmed in a human mixed lymphocyte population whereby the introduction of HSV1716, in the presence of MDMs, resulted in a shift to activated $\mathrm{CD} 4^{+}$and $\mathrm{CD} 8^{+} \mathrm{T}$ cells with an increase in the expression of the co-stimulatory receptor 4-1BB, OX40 (TNF superfamily members) and PD-1 (Supplementary Fig. S7 A\&B). No T cell activation was noted in cultures with uninfected macrophages.

\section{Discussion}

Previous studies have investigated the effect of OV on the survival of malignant cells and the number and activation status of lymphocyte subsets in tumors [24-26]. Although the effect of

OVs on TAMs has not been fully ascertained, primary brain tumors of patients that received intravenous oncolytic reovirus had an unexpected increase in TAMs, suggesting that the role of TAMs in OV therapy should be investigated further [9]. Here we show that TAMs play an important role in mediating the anti-tumor effects of HSV1716. The virus had pronounced effects on the phenotype of these cells and their depletion within the TME reversed the tumoricidal effect of the OV.

TAMs are abundant in most breast tumors [27] and high numbers correlate with reduced patient survival [28-31]. This accords with various studies in mice showing that these cells 
1 stimulate angiogenesis and metastasis in mammary tumors [32, 33]. Macrophages show

2 high plasticity and can move along a continuum between two polarised activation states; 'classically activated', anti-tumor, 'M1-like' TAMs, and 'alternatively activated', tumorpromoting immunosuppressive, 'M2-like TAMs [34]. The latter have been shown to limit tumor responses to various treatments. In our work, we show that although macrophage numbers are stable, there is a marked reduction in the MRC1+ and perivascular macrophages within our tumors after treatment with HSV1716.

In contrast, M1-like TAMs can stimulate cytotoxic T cells by presenting cancer cell antigens to them [3]. This creates a pro-inflammatory tumor microenvironment through the release of cytokines (IL-1, IL-2, IL-6, and IL-12), superoxide anions and nitrogen free radicals. Generally, studies have shown TAMs to be associated with poor patient prognosis and may help facilitate cancer growth $[2,35,36]$. In our study, it is unclear whether the TAM variation is causally linked to the size of the tumours. However like others, we see the re-education of TAMs from an M2-like phenotype to more M1-like is associated with improved survival [37, 38]. Additionally, current evidence suggests that depletion or modification of TAMs alone may result in increased survival in vivo $[39,40]$.

The ability of an OV to reprogram macrophages from an immunosuppressive to an immunostimulatory phenotype opens the potential for OV to be used in conjunction with immunotherapies. Immune checkpoint inhibition involving the blockade of the PD1-PDL1 pathway is currently being promoted in many tumor types. This acts to release an inhibitory break thus allowing $\mathrm{T}$ cells to perform their cytotoxic function. In breast cancer, checkpoint inhibitor monotherapy has a mixed response. This may be due to a failure of activation and migration of $\mathrm{CD}^{+}$cytotoxic T cells within the TME. Given their ability to present antigen and regulate the anti-tumor functions of $\mathrm{T}$ cells, macrophage modification may be key to enhancing this. Indeed, it has been shown that combining PD-1 inhibition (to take the 'brake' off T cells) and CSF-1 receptor blockade (to deplete TAMs within the TME) increases T cell 
1 activation and recruitment within MMTV-PyMT derived MET-1 tumors, thereby increasing the

2 efficacy of the immunotherapy [41].

3

4 However, our data show that macrophages may also play an important role in enhancing OV cytotoxicity by supporting viral amplification. We have previously shown that macrophages can be loaded with OV ex vivo and, upon injection into the circulation of tumor-bearing mice, deliver it to tumors $[16,42]$. In this, we observed an HVS1716 appeared to be amplified by macrophages. Our current work confirms that macrophages not only have the ability to take up HSV1716, enable viral replication and release more HSV1716 particles in vitro, but also re-educate macrophages in the process. Therefore we presume, the lysis of cancer cells by HSV1716 is mediated by direct effects on cancer cells, resulting in immunogenic cell death, and indirect effects on T cells, via TAMs, in the TME.

The ability of HSV1716 to reduce the growth and spread of cancer in our mouse mammary models demonstrates that this may be useful for the treatment of breast cancer and warrants clinical evaluation. HSV1716 is reported to have a good safety profile in non breast cancer early phase clinical trials $[12,13]$ and lends well to seamlessly moving towards translating this research from the bench to the bedside of patients with breast cancer. Our findings indicate that the efficacy of treatments such as checkpoint inhibitors, which require activated T cells to be present in tumors, may be enhanced when used in conjunction with HSV1716.

Previous studies have demonstrated the ability of macrophages to support the replication of highly infective viruses such as the RNA viruses, influenza [43] and simian immunodeficiency virus [44]. We believe we are the first to describe that cancer-killing OV replication occurs within macrophages and that this may enhance virotherapy. Herein, we show that PCNA expression by macrophages supports the replication of HSV1716 in a PCNA-dependent manner. PCNA is an essential component of the "replication and repair" machinery of cells but also shown to be involved in the HSV replication cycle $[45,46]$. It has 
1 been proposed that Neurovirulence Factor ICP34.5 is needed to allow PCNA mediated viral

2 replication in non-dividing cells, with studies showing ICP34.5 deleted HSV strains are avirulent in non-dividing central nervous system neuronal lines [21, 47]. In tumor cells, PCNA is already "switched on" for cellular DNA replication and ICP34.5 is not required to initiate viral replication [45]. Studies comparing the replication of wild type and ICP34.5 deleted HSV1 in Vero (African Green Monkey kidney epithelial) cells demonstrated that viral replication was inhibited when PCNA expression had been knocked down [46]. The implications of this are that PCNA plays a role in HSV DNA replication and that this might be independent of ICP34.5. In untreated breast cancer, high numbers of PCNA-positive TAMs correlate with an immunosuppressive TME and with poor patient prognosis [48-50]. In our in vitro work, exposure of macrophages to tumor-conditioned medium increased their intracellular expression of PCNA suggesting that cancer cells may stimulate TAMs to express a phenotype that supports viral replication. It remains to be seen whether patients with high numbers of PCNA-expressing TAMs respond better to HSV1716 than those with low numbers. If so, this could be a new way to stratify patients for such form of OV therapy.

\section{Acknowledgements}

Thank you to Prof Johanna Joyce (MSKCC, USA) and Dr Jessalyin Ubellacker (University of Harvard, USA) for providing the TS1 and E0771 cell lines respectively. AK \& MM would like to thank Virttu Biologics and CRUK (grant reference: C25574/A24321) and Sheffield Teaching Hospitals Charity (grant reference: 12053) and Team Verrico (grant reference: MS/149394) for their support for various aspects of this work. This project has also received funding from the European Union's Horizon 2020 research and innovation programme under the Marie Sklodowska-Curie grant agreement number 872860-PRISAR2. We are also grateful to Virttu Biologics for providing the HSV1716 for this work. 


\section{References}

1. Sharma P. and Allison J.P., The future of immune checkpoint therapy. Science, 2015. 348(6230): p. 56-61.

2. Lewis C.E., Harney A.S., and Pollard J.W., The Multifaceted Role of Perivascular Macrophages in Tumors. Cancer Cell, 2016. 30(2): p. 365.

3. Williams C.B., Yeh E.S., and Soloff A.C., Tumor-associated macrophages: unwitting accomplices in breast cancer malignancy. NPJ Breast Cancer, 2016. 2.

4. Kioi M., Vogel H., Schultz G., Hoffman R.M., Harsh G.R., and Brown J.M., Inhibition of vasculogenesis, but not angiogenesis, prevents the recurrence of glioblastoma after irradiation in mice. J Clin Invest, 2010. 120(3): p. 694-705.

5. Hughes R., Qian B.Z., Rowan C., Muthana M., Keklikoglou I., Olson O.C., et al., Perivascular M2 Macrophages Stimulate Tumor Relapse after Chemotherapy. Cancer Res, 2015. 75(17): p. 3479-91.

6. Dijkgraaf E.M., Heusinkveld M., Tummers B., Vogelpoel L.T., Goedemans R., Jha V., et al., Chemotherapy alters monocyte differentiation to favor generation of cancersupporting M2 macrophages in the tumor microenvironment. Cancer Res, 2013. 73(8): p. 2480-92.

7. Ribas A., Dummer R., Puzanov I., VanderWalde A., Andtbacka R.H.I., Michielin O., et al., Oncolytic Virotherapy Promotes Intratumoral T Cell Infiltration and Improves Anti-PD-1 Immunotherapy. Cell, 2017. 170(6): p. 1109-1119 e10.

8. Bourgeois-Daigneault M.C., Roy D.G., Aitken A.S., El Sayes N., Martin N.T., Varette O., et al., Neoadjuvant oncolytic virotherapy before surgery sensitizes triple-negative breast cancer to immune checkpoint therapy. Sci Transl Med, 2018. 10(422).

9. Samson A., Scott K.J., Taggart D., West E.J., Wilson E., Nuovo G.J., et al., Intravenous delivery of oncolytic reovirus to brain tumor patients immunologically primes for subsequent checkpoint blockade. Sci Transl Med, 2018. 10(422).

10. Andtbacka R.H., Kaufman H.L., Collichio F., Amatruda T., Senzer N., Chesney J., et al., Talimogene Laherparepvec Improves Durable Response Rate in Patients With Advanced Melanoma. J Clin Oncol, 2015. 33(25): p. 2780-8.

11. Benencia F., Courreges M.C., Conejo-Garcia J.R., Mohamed-Hadley A., Zhang L., Buckanovich R.J., et al., HSV oncolytic therapy upregulates interferon-inducible chemokines and recruits immune effector cells in ovarian cancer. Mol Ther, 2005. 12(5): p. 789-802.

12. Streby K.A., Geller J.I., Currier M.A., Warren P.S., Racadio J.M., Towbin A.J., et al., Intratumoral Injection of HSV1716, an Oncolytic Herpes Virus, Is Safe and Shows Evidence of Immune Response and Viral Replication in Young Cancer Patients. Clin Cancer Res, 2017. 23(14): p. 3566-3574.

13. Mace A.T., Ganly I., Soutar D.S., and Brown S.M., Potential for efficacy of the oncolytic Herpes simplex virus 1716 in patients with oral squamous cell carcinoma. Head Neck, 2008. 30(8): p. 1045-51.

14. Shree T., Olson O.C., Elie B.T., Kester J.C., Garfall A.L., Simpson K., et al., Macrophages and cathepsin proteases blunt chemotherapeutic response in breast cancer. Genes Dev, 2011. 25(23): p. 2465-79.

15. Ranjan A., Gupta P., and Srivastava S.K., Penfluridol: An Antipsychotic Agent Suppresses Metastatic Tumor Growth in Triple-Negative Breast Cancer by Inhibiting Integrin Signaling Axis. Cancer Res, 2016. 76(4): p. 877-90.

16. Muthana M., Giannoudis A., Scott S.D., Fang H.Y., Coffelt S.B., Morrow F.J., et al., Use of macrophages to target therapeutic adenovirus to human prostate tumors. Cancer Res, 2011. 71(5): p. 1805-15.

17. Huber S., Hoffmann R., Muskens F., and Voehringer D., Alternatively activated macrophages inhibit T-cell proliferation by Stat6-dependent expression of PD-L2. Blood, 2010. 116(17): p. 3311-20. 
18. Muthana M., Hawtree S., Wilshaw A., Linehan E., Roberts H., Khetan S., et al., C5orf30 is a negative regulator of tissue damage in rheumatoid arthritis. Proc Natl Acad Sci U S A, 2015. 112(37): p. 11618-23.

19. Baer A. and Kehn-Hall K., Viral concentration determination through plaque assays: using traditional and novel overlay systems. J Vis Exp, 2014(93): p. e52065.

20. Thomas D.L. and Fraser N.W., HSV-1 therapy of primary tumors reduces the number of metastases in an immune-competent model of metastatic breast cancer. Mol Ther, 2003. 8(4): p. 543-51.

21. Kennedy P.G., Gairns J., and MacLean A.R., Replication of the herpes simplex virus type 1 RL 1 mutant 1716 in primary neuronal cell cultures--possible relevance to use as a viral vector. J Neurol Sci, 2000. 179(S 1-2): p. 108-14.

22. van Rooijen N. and Sanders A., Elimination, blocking, and activation of macrophages: three of a kind? J Leukoc Biol, 1997. 62(6): p. 702-9.

23. Denton N.L., Chen C.Y., Hutzen B., Currier M.A., Scott T., Nartker B., et al., Myelolytic Treatments Enhance Oncolytic Herpes Virotherapy in Models of Ewing Sarcoma by Modulating the Immune Microenvironment. Mol Ther Oncolytics, 2018. 11: p. 62-74.

24. Tan D.Q., Zhang L., Ohba K., Ye M., Ichiyama K., and Yamamoto N., Macrophage response to oncolytic paramyxoviruses potentiates virus-mediated tumor cell killing. Eur J Immunol, 2016. 46(4): p. 919-28.

25. Denton N.L., Chen C.Y., Scott T.R., and Cripe T.P., Tumor-Associated Macrophages in Oncolytic Virotherapy: Friend or Foe? Biomedicines, 2016. 4(3).

26. Denton NL C.C., Hutzen B, Leddon J, Wang PY, Scott T, Currier M, Eubank T, Cripe TP, Tumor Associated Macrophages Mitigate Oncolytic Herpes Simplex Virus Efficacy in Part Through TGF- $\beta$ Signaling. Molecular Therapy, 2016. Volume 24, Supplement 1: p. pS206-S207.

27. Kelly C.A., Ward C., Stenton S.C., Hendrick D.J., and Walters E.H., Assessment of pulmonary macrophage and neutrophil function in sequential bronchoalveolar lavage aspirates in sarcoidosis. Thorax, 1988. 43(10): p. 787-91.

28. Zhao X., Qu J., Sun Y., Wang J., Liu X., Wang F., et al., Prognostic significance of tumor-associated macrophages in breast cancer: a meta-analysis of the literature. Oncotarget, 2017. 8(18): p. 30576-30586.

29. DeNardo D.G., Brennan D.J., Rexhepaj E., Ruffell B., Shiao S.L., Madden S.F., et al., Leukocyte complexity predicts breast cancer survival and functionally regulates response to chemotherapy. Cancer Discov, 2011. 1(1): p. 54-67.

30. Shabo I., Stal O., Olsson H., Dore S., and Svanvik J., Breast cancer expression of CD163, a macrophage scavenger receptor, is related to early distant recurrence and reduced patient survival. Int J Cancer, 2008. 123(4): p. 780-6.

31. Kurahara H., Shinchi H., Mataki Y., Maemura K., Noma H., Kubo F., et al., Significance of M2-polarized tumor-associated macrophage in pancreatic cancer. J Surg Res, 2011. 167(2): p. e211-9.

32. Lin H., Chen C., and Chen B.D., Resistance of bone marrow-derived macrophages to apoptosis is associated with the expression of $X$-linked inhibitor of apoptosis protein in primary cultures of bone marrow cells. Biochem J, 2001. 353(Pt 2): p. 299-306.

33. Lin E.Y., Li J.F., Gnatovskiy L., Deng Y., Zhu L., Grzesik D.A., et al., Macrophages regulate the angiogenic switch in a mouse model of breast cancer. Cancer Res, 2006. 66(23): p. 11238-46.

34. Yang M., McKay D., Pollard J.W., and Lewis C.E., Diverse Functions of Macrophages in Different Tumor Microenvironments. Cancer Res, 2018. 78(19): p. 5492-5503.

35. Jakeman P.G., Hills T.E., Fisher K.D., and Seymour L.W., Macrophages and their interactions with oncolytic viruses. Curr Opin Pharmacol, 2015. 24: p. 23-9.

36. Murdoch C., Muthana M., Coffelt S.B., and Lewis C.E., The role of myeloid cells in the promotion of tumour angiogenesis. Nat Rev Cancer, 2008. 8(8): p. 618-31. 
37. Tariq M., Zhang J., Liang G., Ding L., He Q., and Yang B., Macrophage Polarization: Anti-Cancer Strategies to Target Tumor-Associated Macrophage in Breast Cancer. J Cell Biochem, 2017. 118(9): p. 2484-2501.

38. Georgoudaki A.M., Prokopec K.E., Boura V.F., Hellqvist E., Sohn S., Ostling J., et al., Reprogramming Tumor-Associated Macrophages by Antibody Targeting Inhibits Cancer Progression and Metastasis. Cell Rep, 2016. 15(9): p. 2000-11.

39. Fritz J.M., Tennis M.A., Orlicky D.J., Lin H., Ju C., Redente E.F., et al., Depletion of tumor-associated macrophages slows the growth of chemically induced mouse lung adenocarcinomas. Front Immunol, 2014. 5: p. 587.

40. Poh A.R. and Ernst M., Targeting Macrophages in Cancer: From Bench to Bedside. Front Oncol, 2018. 8: p. 49.

41. Peranzoni E., Lemoine J., Vimeux L., Feuillet V., Barrin S., Kantari-Mimoun C., et al., Macrophages impede CD8 T cells from reaching tumor cells and limit the efficacy of anti-PD-1 treatment. Proc Natl Acad Sci U S A, 2018. 115(17): p. E4041-E4050.

42. Muthana M., Rodrigues S., Chen Y.Y., Welford A., Hughes R., Tazzyman S., et al., Macrophage delivery of an oncolytic virus abolishes tumor regrowth and metastasis after chemotherapy or irradiation. Cancer Res, 2013. 73(2): p. 490-5.

43. Cline T.D., Beck D., and Bianchini E., Influenza virus replication in macrophages: balancing protection and pathogenesis. J Gen Virol, 2017. 98(10): p. 2401-2412.

44. Williams K., Schwartz A., Corey S., Orandle M., Kennedy W., Thompson B., et al., Proliferating cellular nuclear antigen expression as a marker of perivascular macrophages in simian immunodeficiency virus encephalitis. Am J Pathol, 2002. 161(2): p. 575-85.

45. Harland J., Dunn P., Cameron E., Conner J., and Brown S.M., The herpes simplex virus (HSV) protein ICP34.5 is a virion component that forms a DNA-binding complex with proliferating cell nuclear antigen and HSV replication proteins. J Neurovirol, 2003. 9(4): p. 477-88.

46. Sanders I., Boyer M., and Fraser N.W., Early nucleosome deposition on, and replication of, HSV DNA requires cell factor PCNA. J Neurovirol, 2015. 21(4): p. 35869.

47. Ward S.L., Scheuner D., Poppers J., Kaufman R.J., Mohr I., and Leib D.A., In vivo replication of an ICP34.5 second-site suppressor mutant following corneal infection correlates with in vitro regulation of elF2 alpha phosphorylation. J Virol, 2003. 77(8): p. 4626-34.

48. Campbell M.J., Tonlaar N.Y., Garwood E.R., Huo D., Moore D.H., Khramtsov A.I., et al., Proliferating macrophages associated with high grade, hormone receptor negative breast cancer and poor clinical outcome. Breast Cancer Res Treat, 2011. 128(3): p. 703-711.

49. Campbell M.J., Wolf D., Mukhtar R.A., Tandon V., Yau C., Au A., et al., The prognostic implications of macrophages expressing proliferating cell nuclear antigen in breast cancer depend on immune context. PLoS One, 2013. 8(10): p. e79114.

50. Mukhtar R.A., Moore A.P., Nseyo O., Baehner F.L., Au A., Moore D.H., et al., Elevated PCNA + tumor-associated macrophages in breast cancer are associated with early recurrence and non-Caucasian ethnicity. Breast Cancer Res Treat, 2011. 130(2): p. 635-44. 
Figures legends:

4

Figure 1: HSV1716 treatment inhibits primary mammary tumor growth and metastatic spread. PyMT-TS1, EO771 and 4T1 cells were implanted into the $4^{\text {th }}$ mammary fat pad of immuno-competent syngeneic female mice. When tumors reached $\sim 150 \mathrm{~mm}^{3}$, mice received intravenous HSV1716 (dose $1 \times 10^{6}$ PFU) or PBS. Tumor volume was assessed by caliper measurements in vivo and pulmonary metastases were measured postmortem by $\mathrm{H} \& \mathrm{E}$ staining. HSV1716 (grey line) significantly reduced (A) mammary tumor growth and (B) subsequent development of lung metastases in all three models. Data shown are mean \pm SEM, $n=10$ mice/treatment group and statistical significance analyzed using multiple $t$ tests where ${ }^{*}=P<0.05,{ }^{* *}=P<0.01,{ }^{* *}=P<0.001,{ }^{* * * *}=P<0.0001$ compared to control (untreated cells).

\section{Figure 2: HSV1716 treatment prevents tumor spread in a model of metastatic seeding.} Luciferase labeled $4 \mathrm{~T} 1$ cells were injected into the left ventricles of female BALB/c mice. At day 5, mice were treated with PBS, HSV1716 ( 1 X10 ${ }^{7}$ PFU, single dose) or HSV1716 $\left(1 \times 10^{7}\right.$ on days $5,7,9)$. (A) Schematic representation of the treatment schedule is shown. (B) Representative images of metastatic burden in different treatment groups at day 20. Tumor burden was inhibited in the OV treated group with no disease observed in the 3-dose group at the end of experiment. (C) Overall survival was also increased in all mice that received virus. There is a statistically significant reduction in the survival between the control (phosphate-buffered saline, PBS, treated) and the group that received 3 doses of HSV1716 $(p=0.0002, C l 15.49-35.18)$ and between the OVx1 and OVx3 group $(p=0.01, C l 5.401$ to 31.93). (D) Burden of metastases was calculated as the percentage of the organ with metastatic involvement; this was calculated as an average between 4 slides in 2-4 different sections of organ, over $100 \mu m$ apart. Data shown are mean \pm SEM, $n=6$ animals. Statistical significance was assessed by one-way ANOVA where ${ }^{*} P<0.05$. 
1 Figure 3. HSV1716 treatment induces inflammation within the tumour

2 microenvironment (TME). PyMT tumors were grown in syngeneic female FVB mice and

3 randomized into 2 treatment arms, control (PBS) and intravenous HSV1716 treatment $(n=5$ 10 per arm). (A) RNA was isolated from tumors and analysed using the NanoString nCounter ${ }^{\mathrm{TM}}$ murine Pan-Cancer Immune Profiling Panel. Volcano plots show genes that were up regulated or downregulated following IV HSV1716 treatment compared to tumors in PBS-treated mice $(n=3)$. The data were processed and analysed using nSolver ${ }^{\text {TM }}$ Analysis Software, using the Advanced Analysis module. (B) Flow cytometric data from these enzymatically digested tumor specimens are shown from $n=10$ mice per group. Only viable cells (UV-) were used in this analysis. Each immune cell population was gated upon based on CD45 expression and the respective immune cell marker. This shows changes in the percentage of infiltrating immune cells (myeloid cells $\left(\mathrm{F}_{480^{+}}\right)$, monocytes $\left(\mathrm{Ly}_{6 \mathrm{C}} \mathrm{C}^{+}\right.$, neutrophils $\left(\mathrm{Ly}_{6 \mathrm{G}}{ }^{+}\right)$, $\mathrm{T}$ cells $\left(\mathrm{CD}^{+,} \mathrm{CD}^{+}, \mathrm{CD} 8^{+}\right)$) within the TME. (C) Immunofluorescent staining of respective tumors ( 5 fields per view per slide) confirmed that these $\mathrm{T}$ cells become activated in response to HSV1716 treatment, as illustrated by increased expression of IFNg and PD1 and (D) a decrease in the prevalence of CD4 ${ }^{+} \mathrm{FOXP} 3^{+}$immunoregulatory $\mathrm{T}$ (Treg) cells. Data shown are mean \pm SEM, analysed by Student $t$ tests where $p<0.05$.

\section{Figure 4. HSV1716 treatment reprograms TAMs to become less perivascular and more} pro-inflammatory. (A) Sections of primary tumors, derived from the PyMT-TS1 model, treated with intravenous HSV1716 (OV) or PBS show a significant decrease in the number of perivascular $\left(\mathrm{CD} 31^{+}\right)$macrophages after HSV1716 treatment $(\mathrm{n}=10$ animals). These were quantified by only counting the F4/80+ cells in direct contact with CD31+ cells. (B) HSV1716 treatment also causes a shift in macrophage phenotype with a down regulation in $\mathrm{F} 4 / 80^{+} \mathrm{MRC}^{+}$cells and a significant increase in presence of $\mathrm{F} 4 / 80^{+} \mathrm{IL}-12^{+}$and $\mathrm{F} 4 / 80^{+} \mathrm{NOS}^{+}$ cells. Images were taken using the Nikon A1 confocal microscope and scored using FIJI image $\mathrm{J}$ software. Co-localization between F4/80 and MRC1, IL-12 or iNOS was determined and quantified using the Cell Counter tool from ImageJ (Fiji) [National Institutes of Health 
1 (NIH), Bethesda, MD, USA] and 5 randomly selected fields of view were imaged per tumor.

2 Data shown are mean \pm SEM, analysed by student $t$ tests where $p<0.05$.

3

4 Figure 5. Monocyte-derived macrophages (MDMs) are permissive to HSV1716

5 infection and replication. (A) Representative sections from primary PyMT TS1 tumors in control (PBS) and HSV1716-treated mice showed co-localisation of $\mathrm{F} 4 / 80^{+}$macrophages (green) and HSV1716 (white). This was quantified in $n=10$ animals, 5 fields per view. (B) MDMs were infected with HSV1716 expressing GFP at MOI 5. In vitro infection was assessed by flow cytometry (left image) and fluorescence (right image). (C) Supernatants taken from these cultures were assessed by the plaque-forming assay on Vero cells and an increase in viral titres over time suggested active viral replication within these macrophages. (D) qPCR analysis of MDMs at $24 \mathrm{~h}$ post infection resulted in the mRNA expression of the viral early-ICP0 (Left), mid-ICP8 (centre) and late-gB (right) replication genes. Data shown are mean \pm SEM, analysed by student $t$ test where $p<0.05, n=5$ independent experiments.

\section{Figure 6. PCNA mediates HSV1716 replication in monocyte-derived macrophages} (MDMs). Female FVB tumor-bearing mice (PyMT-TS1) received a single dose of intravenous HSV1716 at $10^{6}$ PFU (OV) or PBS (control). (A) PCNA expression by TAMs (arrowed) is significantly upregulated in response to HSV1716 at day 9 post treatment $(n=5$ mice per group). (B) In vitro studies confirmed that PCNA expression at both the RNA and protein level by MDMs significantly increases after infection. (C) Accell siRNA was used to knock down PCNA (PCNA ${ }^{\mathrm{KD}}$ ) within MDMs. The human breast cancer cell line MDA-MB-231 was treated with supernatants from MDMs infected with HSV1716 (with or without PCNA ${ }^{\mathrm{KD}}$ ) at different MOIs. At 72 hours we see inhibition of viral induced cell death in cultures where PCNA is knocked down. (D) PCNA knockdown also suppressed replication of virus infected cells (MOI 5), as shown by qPCR of MDMs at 72 hours post infection with suppression of early-ICPO (Left), mid-ICP8 (centre) and late-gB (right) viral replication genes. Key: OV = 
1 oncolytic virus and $P C N A^{K D}+O V=P C N A$ knockdown and OV. Data shown are mean $\pm \mathrm{SEM}$

2 and analyzed using a one-way ANOVA where $p<0.05, n=5$ experiment

3

4 Figure 7: HSV1716 cytotoxicity is dependent on macrophages and transforms

5 immunogenically 'cold' tumors to 'hot' tumors. Orthotopically implanted PyMT-TS1

6 tumors were grown immunocompetent syngeneic female FVB mice. Macrophages were

7 eliminated by intravenous administration of clodronate liposomes 48 hours before

8 administration of HSV1716 ( $\mathrm{n}=5$ animals, 1 section, 5 fields per view per section). (A)

9 Clodronate liposomes decrease TAMs within the TME (brighter cells $=\mathrm{F} 480^{+}$cells). This

10 depletion of TAMs attenuated the influence of HSV1716 treatment on the growth of primary

11 tumors (B) and development of pulmonary metastases (C) in vivo. (D) An increase in helper

$12 \mathrm{CD}^{+} \mathrm{T}$ cells and a reduction in cytotoxic $\mathrm{CD}^{+}$cells was observed after HSV1716 treatment

13 (brighter cells). Data shown are mean \pm SEM, one-way ANOVA where $p<0.05, n=5$

14 experiments. 
1 Figures:

Figure 1

A.

Ts1

E0771

4T1
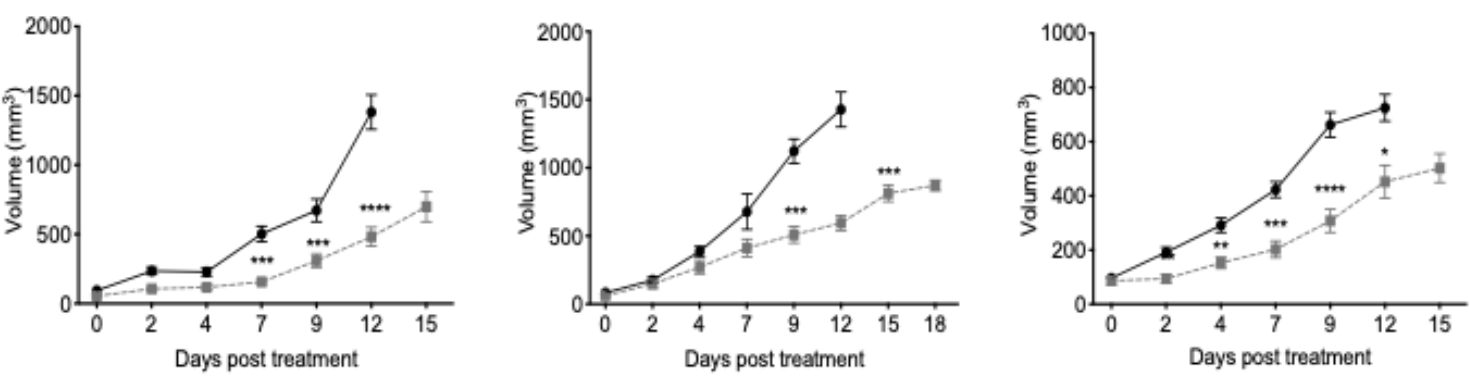

B.

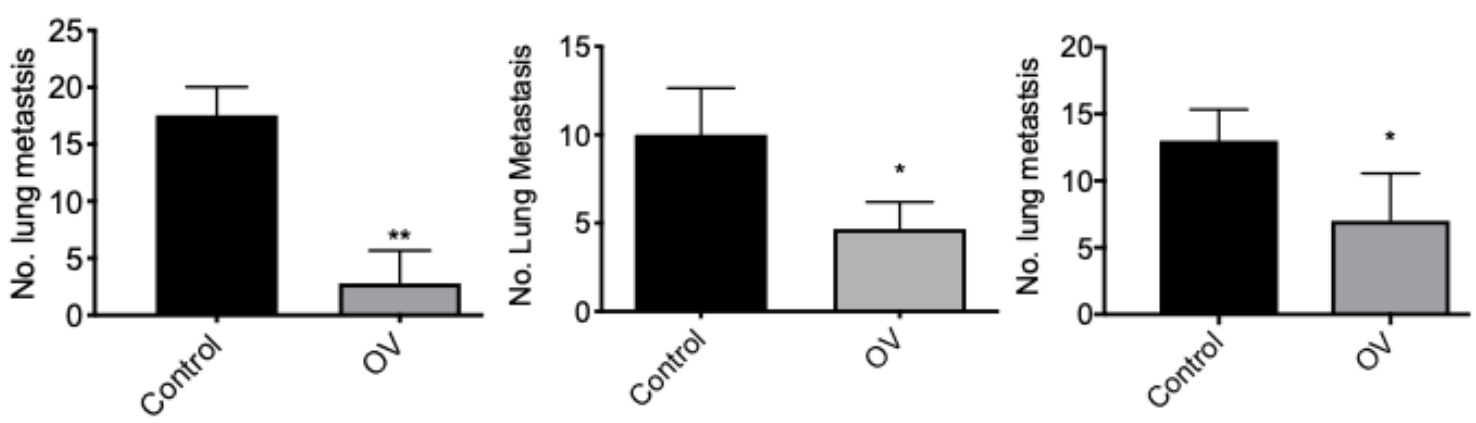




\section{Figure 2}

A.

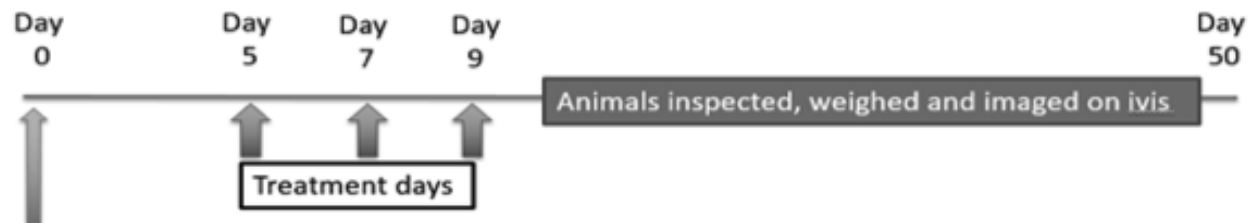

LUC-4t1-BR

cells implanted

via intracardiac

route

B.

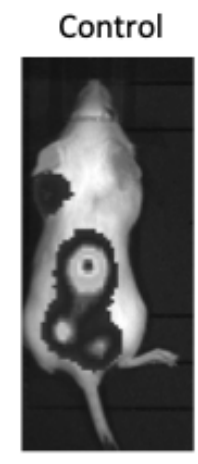

D.

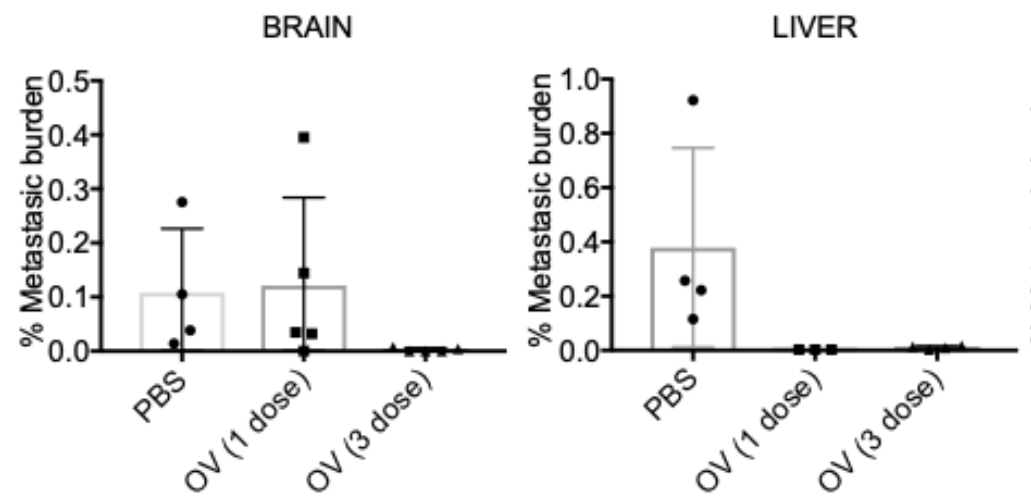

C.

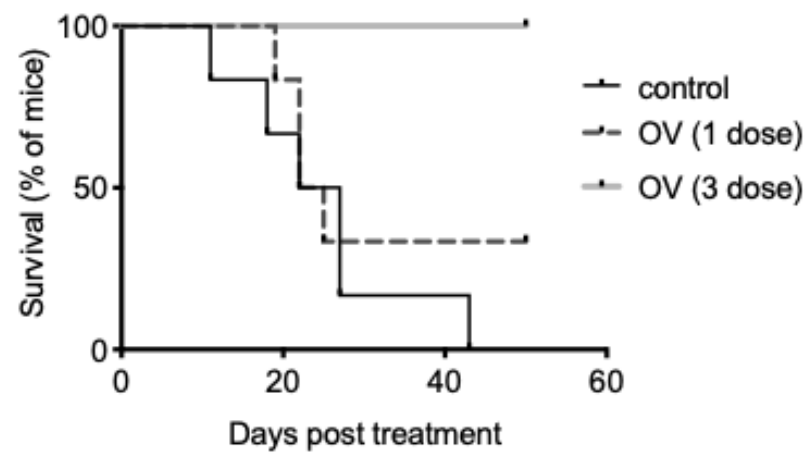




\section{Figure 3}
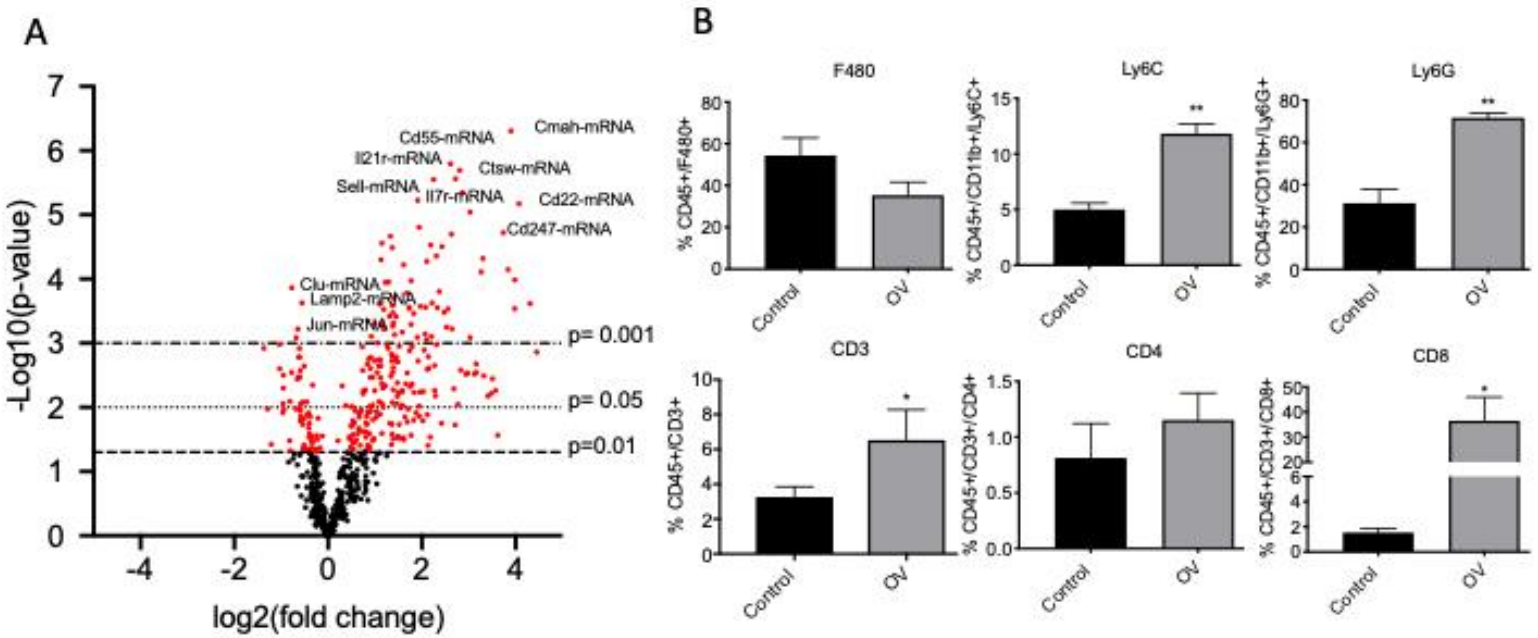

C
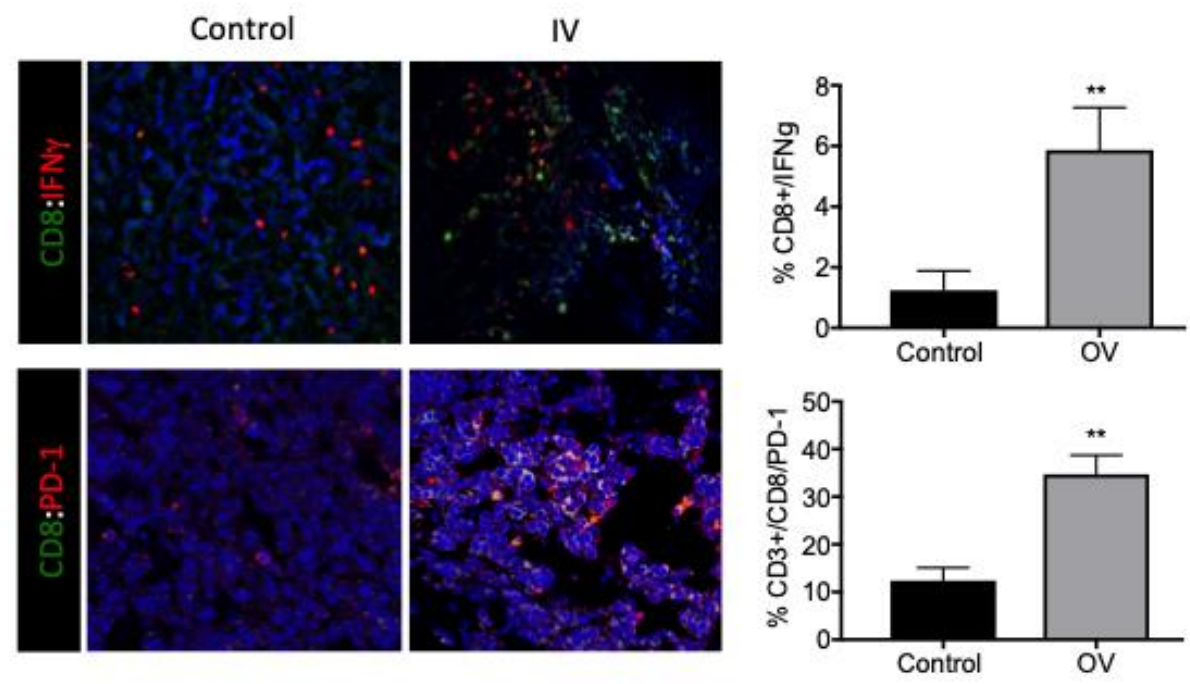

D
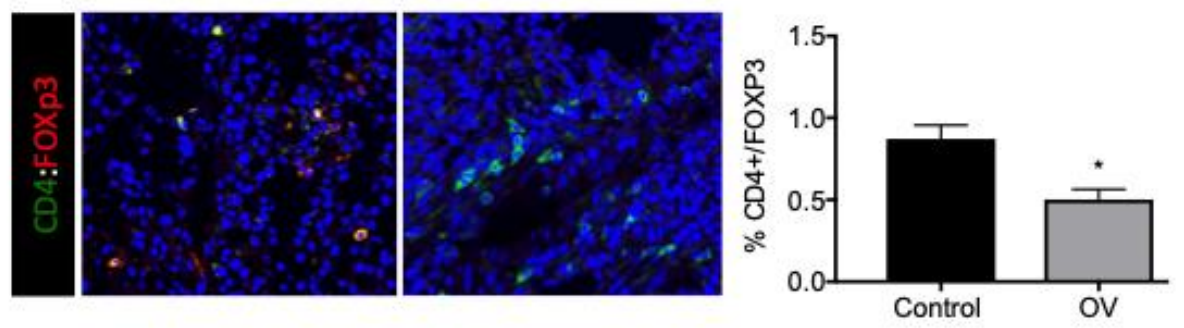
Figure 4
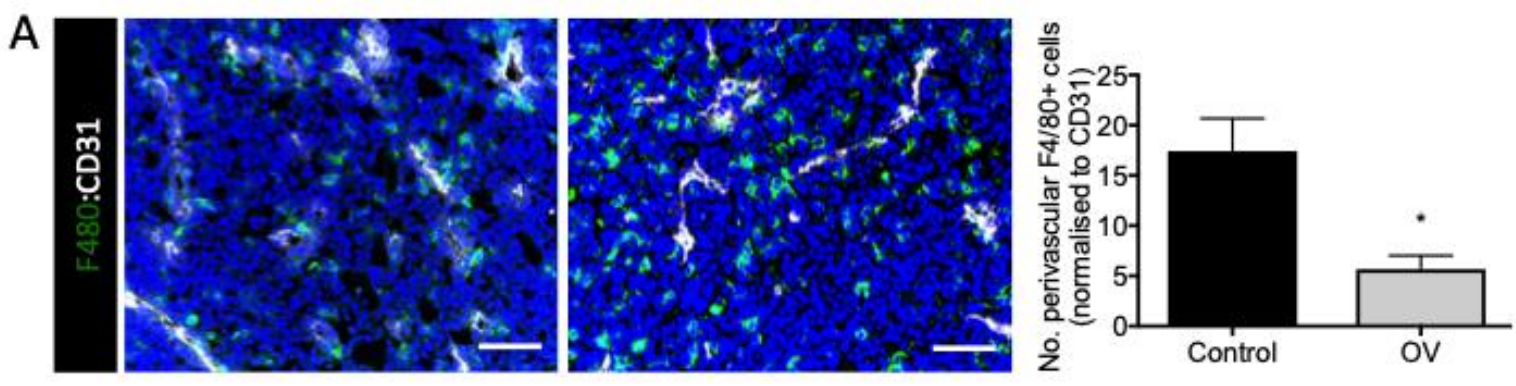

B
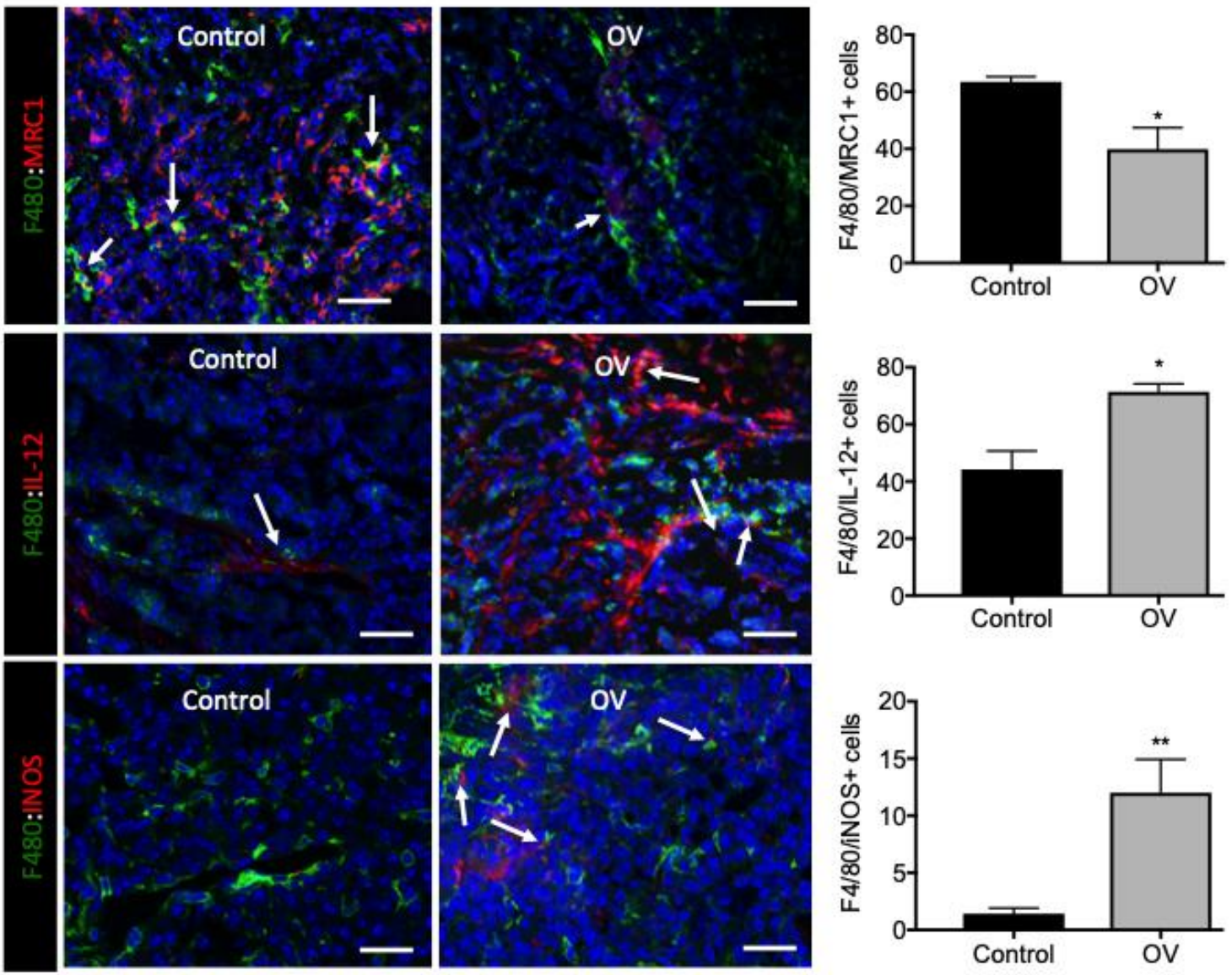
Figure 5
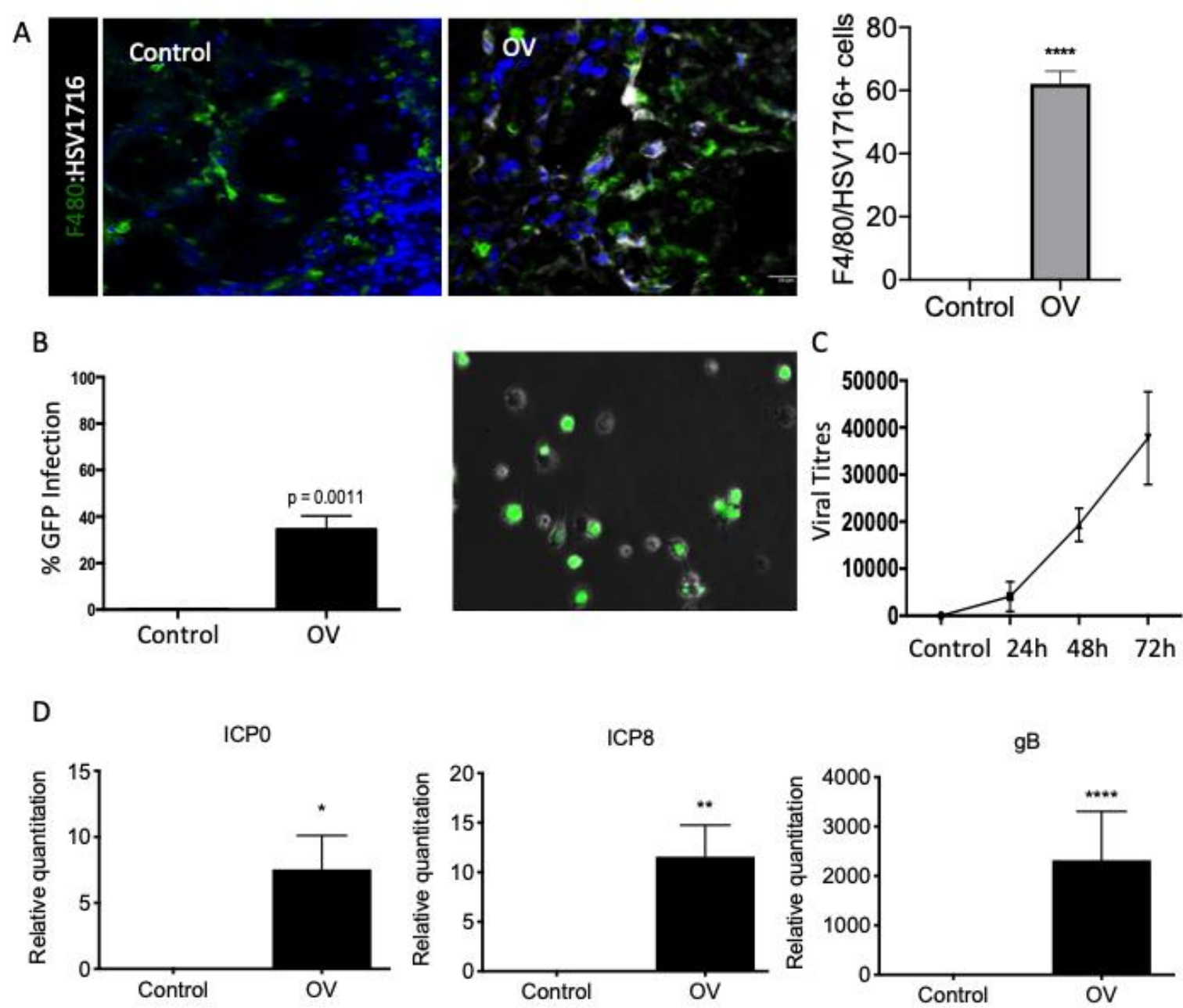
Figure 6

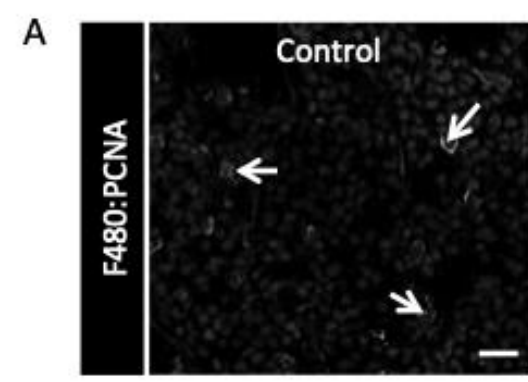

B

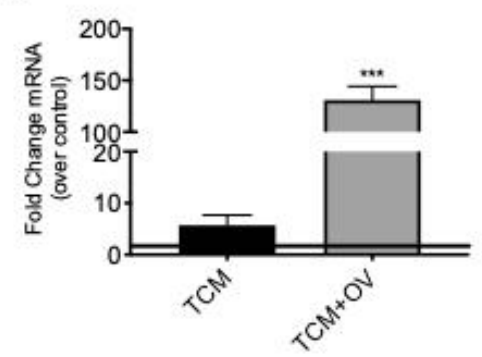

D

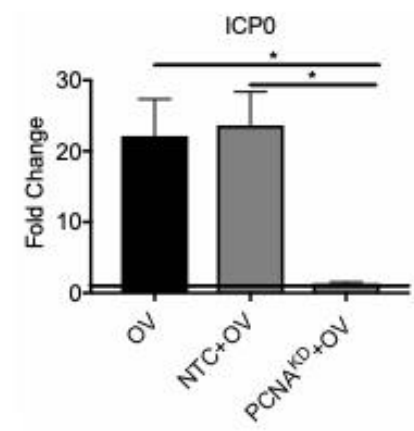

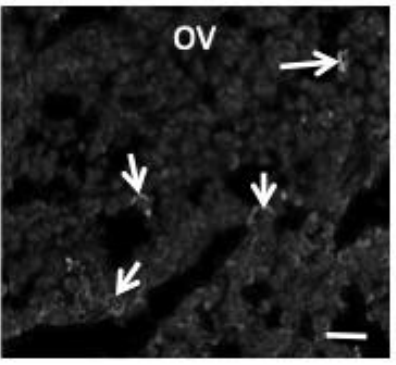

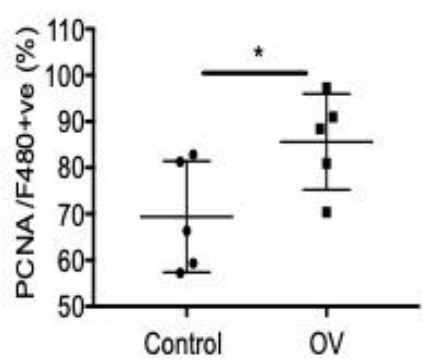

C

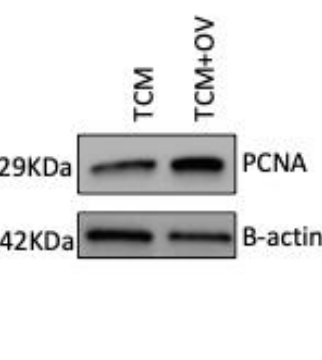

ICP8

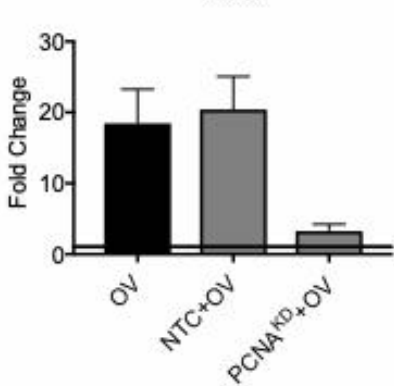

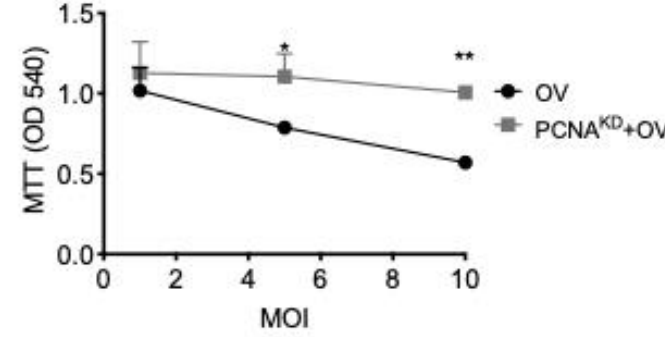

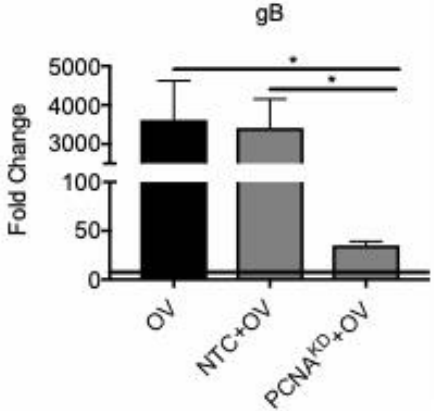


Figure 7
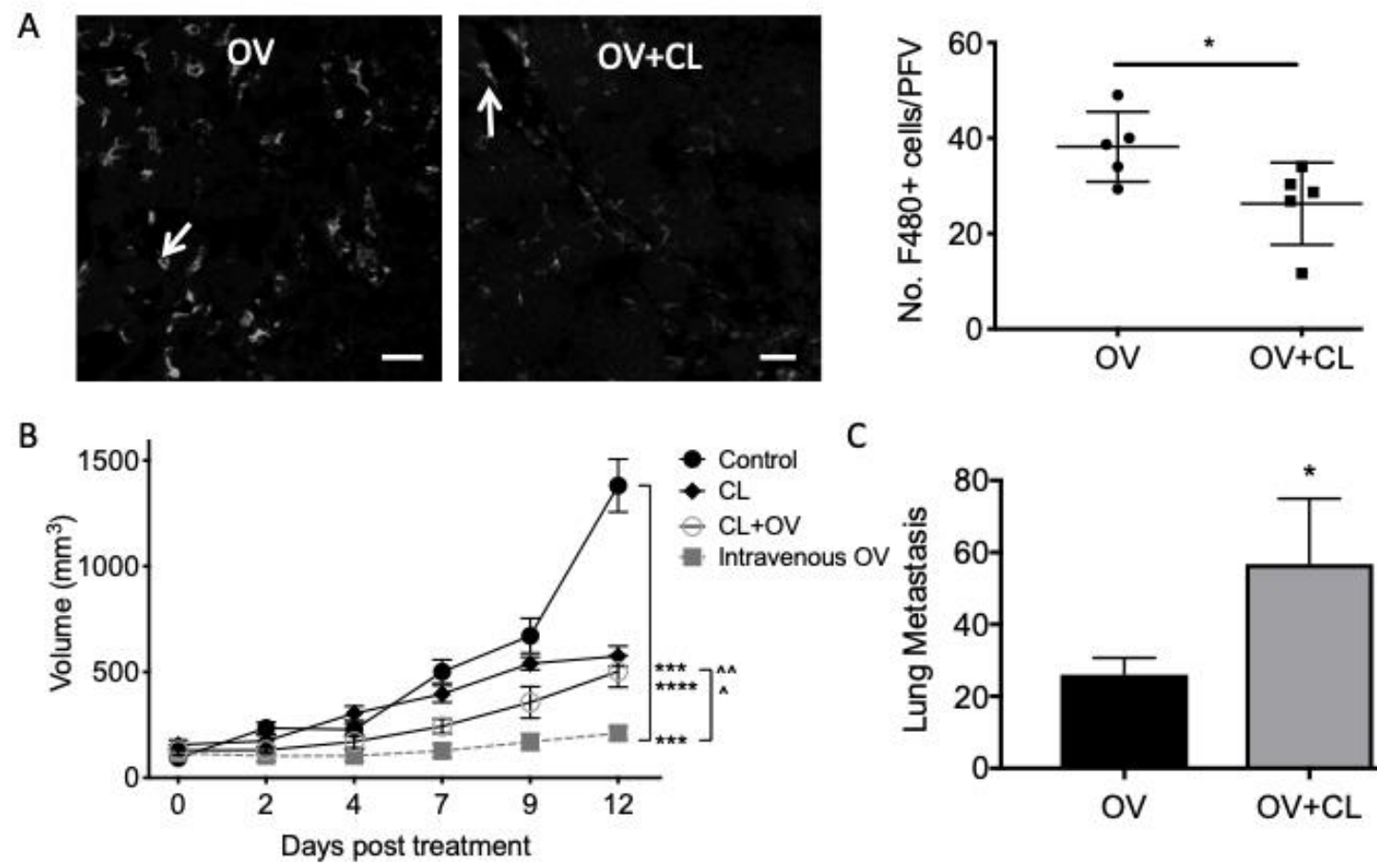

C

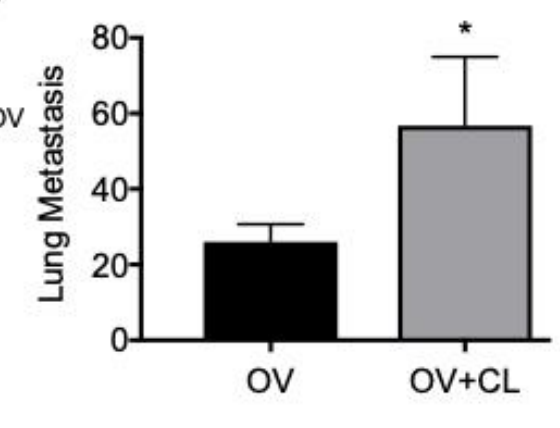

D
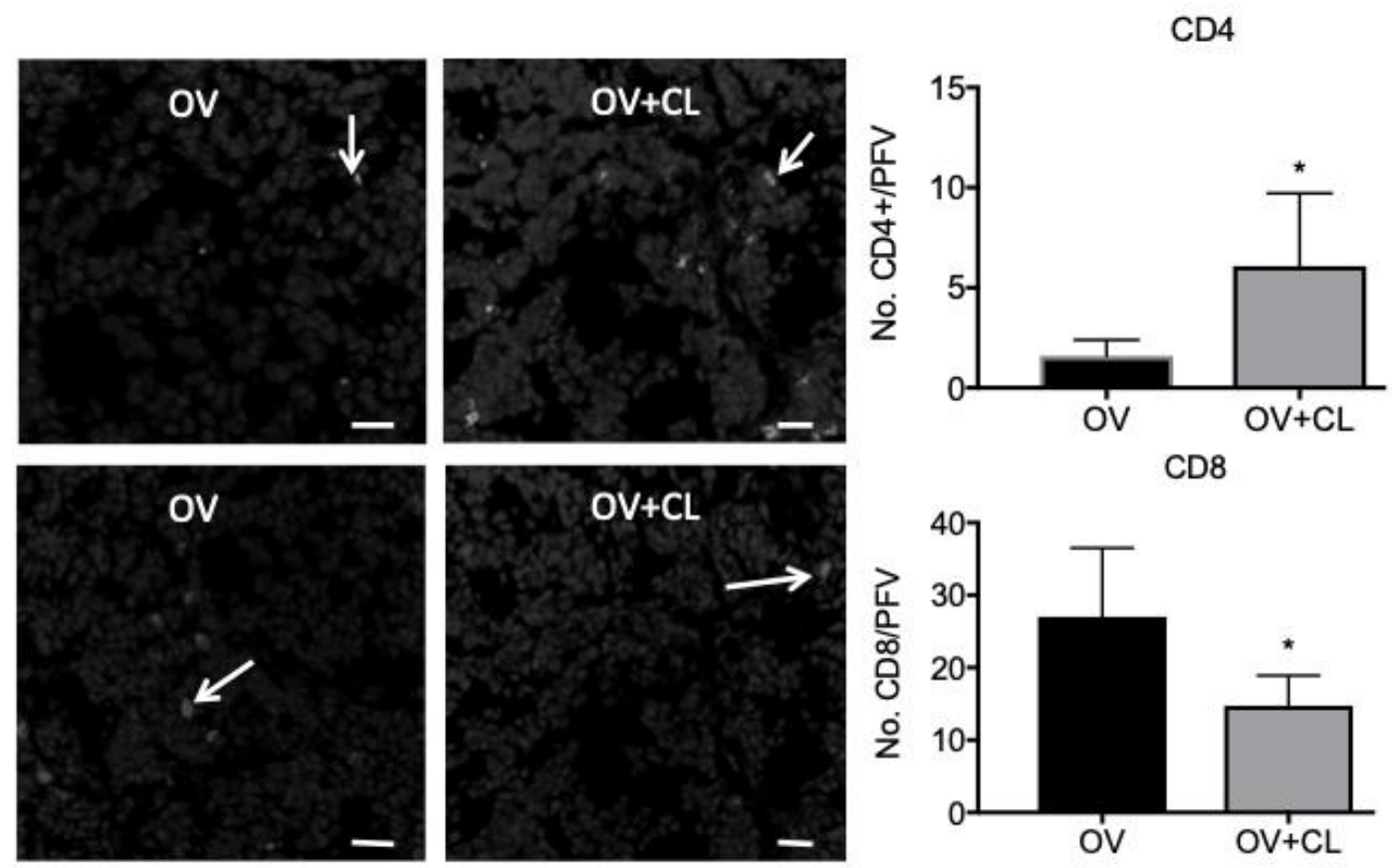